\title{
Microstructural and Diffusion Analysis of Au-Sn Diffusion Couple Layer Undergoing Heat Treatment at Near Eutectic Temperatures
}

\author{
Jidsucha Darayen ${ }^{1, a}$, Panaaek Athichalinthorn ${ }^{2}$, RatchateeTechapiesancharoenkij ${ }^{2}$, \\ Sasawat Mahabunphachai ${ }^{3}$, Chedtha Puncreobutr ${ }^{1}$, Gobboon Lothongkum ${ }^{1}$, \\ and Boonrat Lohwongwatana ${ }^{1, b, *}$
}

1 Innovative Metals Research Unit, Department of Metallurgical Engineering, Faculty of Engineering, Chulalongkorn University, Bangkok, Thailand

2 Department of Materials Engineering, Faculty of Engineering, Kasetsart University, Bangkok, Thailand 3 National Metal and Materials Technology Center (MTEC), National Science and Technology

Development Agency (NSTDA), Pathum Thani, Thailand

E-mail: abeer.chabu@gmail.com, bboonrat@gmail.com (Corresponding authors)

\begin{abstract}
Diffusion couples of pure gold and pure tin were created by mechanical cold rolling method. The couples were isothermally treated at temperatures slightly above and below the eutectic temperature near tin-rich region of the equilibrium phase diagram. Differences in the diffusion behaviors were observed as a function of treatment temperatures below $(473 \mathrm{~K})$ and above $(498 \mathrm{~K})$ the eutectic temperature. At the boundary, it was found that first solid state inter-diffusion was initiated which resulted in local compositional change and solid-state formation of intermetallic compounds (IMCs). As the composition shifts away towards mixing, the growth of the intermetallic phases was monitored as a function of temperature and time. At temperature above the eutectic, there may be a liquid fraction as the interface isothermally melted. The kinetic involves dissolution of $\mathrm{Au}$ atoms into localized tin-rich liquid. At below eutectic temperature, the formation and growth kinetic of phases follows a solid state diffusion mechanism. By investigation the exponent $\mathrm{n}$ values in the growth equation $\mathrm{l}=\mathrm{k}(\mathrm{t} / \mathrm{t} 0) \mathrm{n}$, the values were found to be in between $0.62-0.77$ which implies that the kinetics of IMC formations experiment are controlled by both diffusion and intermetallic reaction. The bonding time was found to be faster and more reliable at bonding temperature slightly above the eutectic.
\end{abstract}

Keywords: Eutectic, Au-Sn, soldering, bonding, electronic soldering, intermetallic.

ENGINEERING JOURNAL Volume 21 Issue 1

Received 28 July 2016

Accepted 2 August 2016

Published 31 January 2017

Online at http://www.engj.org/

DOI:10.4186/ej.2017.21.1.245 


\section{Introduction}

Since lead solders were deemed hazardous by many countries globally, much research focus has been directed upon tin-based solder families. Depending on the bonding temperatures, families of tin-based alloys have been heavily utilized by the electronic and automotive industries [1,2]. Our paper focuses on the binary AuSn alloy system which is extensively used in electronic industry due to its higher melting point than regular $\mathrm{Sn}-\mathrm{Ag}-\mathrm{Cu}$ alloy system as well as its stability at high temperatures. Binary Au-Sn solder system was investigated for temperature and time effects on microstructural change. There have been debates on the formation order of intermetallic phases such as AuSn and $\mathrm{AuSn}_{4}$. Moreover from the manufacturing point of view, most compositions of Au-Sn soldering procedure are considered fluxless owing to the fact that oxide film of metal may not appear in this system [3]. Moreover, Au-Sn alloy generally provides reliable mechanical properties while maintaining thermal stability, relatively good wettability, and good creep resistance [4]. From previous studies [3, 5-7], interdiffusion layer of IMC phases were formed during both soldering and/or annealing processes. An interesting interconnects technology for high temperature applications is solid-liquid interdiffusion (SLID) which is also known as Transient Liquid Phase (TLP) bonding. There are two metals which have different melting temperatures; one is lower and another is higher which will become dissoluted into liquid fraction of the lower melting metal. The formation and bond strength depend on the formation of IMCs. In our case, considering diffusion mechanism, while the bulk (interstitial) diffusion is dominating, gold diffuse into tin faster than vice versa due to the smaller atomic radii of gold element[4].

In the binary Au-Sn system, there are two eutectic compositions at $\mathrm{Au}-29 \% \mathrm{Sn}\left(\mathrm{T}_{\text {eutectic }}=553 \mathrm{~K}\right)$ and $\mathrm{Au}-$ $95.4 \% \mathrm{Sn}\left(\mathrm{T}_{\text {eutectic }}=490 \mathrm{~K}\right)$. Au-Sn phase diagram is shown in Fig. 1. Two eutectic points are located in both tin-rich and gold-rich regions. For most conventional solder, the eutectic Au-Sn compositions are used in high reliability applications especially the gold-rich composition [8-10]. In this phase diagram, there are many varieties of IMC phases [11, 12]. However, of all these phases, $\mathrm{AuSn}, \mathrm{AuSn}_{2}$, and $\mathrm{AuSn}_{4}$ are confirmed by previous studies $[3,4,13]$. These phases appear in the solder microstructure at the room temperature while Au-rich phase and $\mathrm{Au}_{5} \mathrm{Sn}$, could not be completely distinguished as they could be at the resolution limit of conventional techniques [14]. These IMC phases have been different properties in each phase of IMCs in Au-Sn system. Moreover, strength of entire solder stack also depends on the strength of interface amongst IMC phases. For example, the interface between $\mathrm{AuSn}_{2}$ and $\mathrm{AuSn}_{4}$ is brittle and crack usually initiates from this region [15]. There have also been findings of contradicting results on which phases are formed first. There are numerous reports on the initial formation of $\mathrm{AuSn}_{4}$ but at the same time the initial observation of AuSn prior to $\mathrm{AuSn}_{4}$ had also been reported.

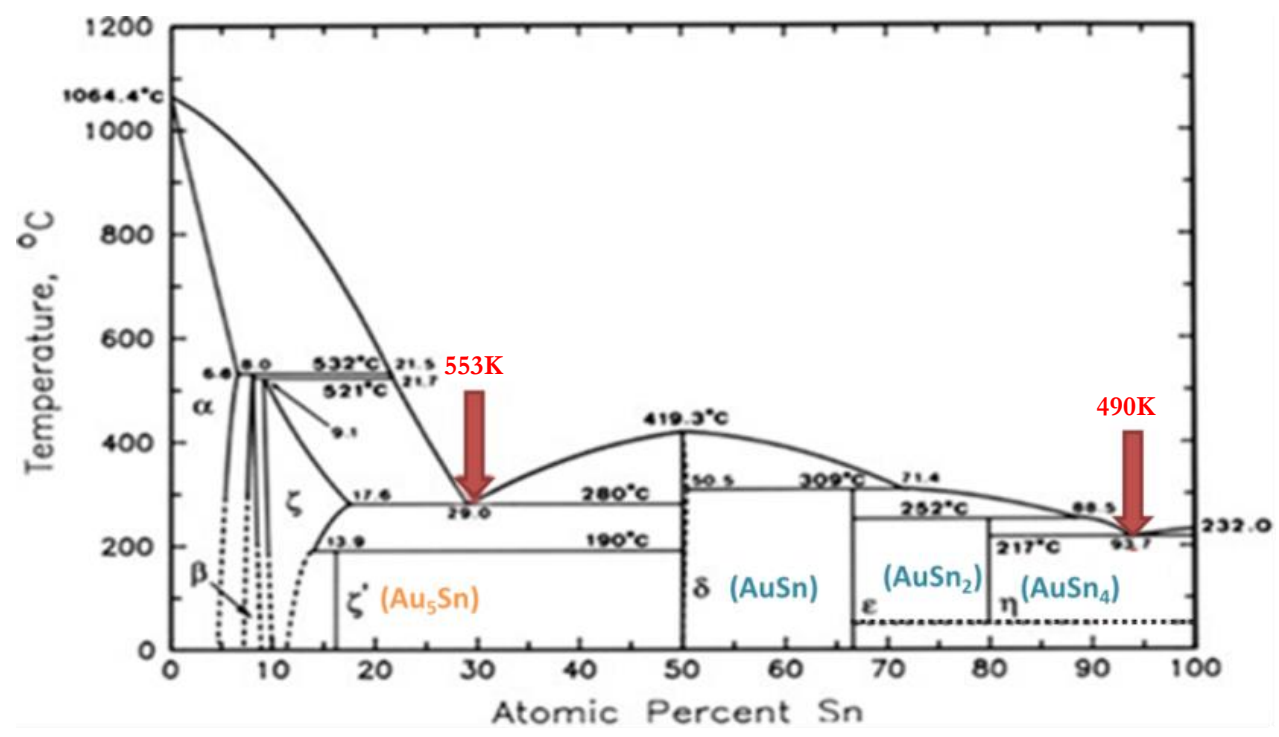

Fig. 1. Equilibrium phase diagram of binary Au-Sn alloy system (adapted from [3]). The two arrows indicate two eutectic points. The experiments in this paper were carried out at above and below the eutectic temperature at the $\mathrm{Sn}$-rich eutectic side. 
For industrial applications, the compositions are focused on the either the Au-rich or Sn-rich compositions. Near Au-rich composition, the following phases have been reported: Au solid solution, $\mathrm{Au}_{10} \mathrm{Sn}$ $(\beta), \mathrm{Au}_{5} \mathrm{Sn}\left(\zeta^{\prime}\right)$, and $\mathrm{AuSn}(\delta)$ phases. The Au-rich solid solution has a maximum solubility of $\mathrm{Sn}$ in a range of 6.1 atomic $\%$ Sn near $805 \mathrm{~K}$. The Sn atoms replace Au atoms substitutionally. In addition, Sn solute may be interstitial atoms between some Au atoms. The crystal structure of Au solid solution is face-centered cubic which is known for more ductility.

For Sn-rich range (more than 50 at $\% \mathrm{Sn}$ ), there are two intermetallic compounds, $\mathrm{AuSn}_{2}(\varepsilon)$ and $\mathrm{AuSn}_{4}$ $(\eta)$, and $\mathrm{Sn}$-rich $\beta$-solid solution phase. The eutectic point on the $\mathrm{Sn}$-rich side is at $95.4 \mathrm{at} \% \mathrm{Sn}$ with a eutectic temperature as low as $505 \mathrm{~K}$. The eutectic phases comprise of $\beta$ solid solution and $\eta\left(\mathrm{AuSn}_{4}\right)$. There is a limit solid solubility of Au atoms inside Sn matrix.

To summarize the mechanical properties based on the crystal structures and phases. There are six important intermetallic (IMC) phases as follows:

- The $\beta$ phase, $\mathrm{Au}_{10} \mathrm{Sn}$, has a hexagonal structure. The structure is stable in the temperature range above $190{ }^{\circ} \mathrm{C}$ up to peritectic temperature at $532^{\circ} \mathrm{C}$. This $\beta$ phase has relatively higher modulus and high Vickers hardness. As a result, the fracture reveals brittleness nature of this hexagonal structure.

- The $\zeta$ phase, $\mathrm{Au}_{17} \mathrm{Sn}_{3}$, also has a hexagonal structure which is more relaxing than the $\beta$ phase, $\mathrm{Au}_{10} \mathrm{Sn}$, in term of compositional range that can accommodate tin atoms from 12 to 16 at $\% \mathrm{Sn}$ at temperature range $220-225^{\circ} \mathrm{C}$. This temperature range is near bonding and processing temperatures in many soldering processes. $\mathrm{Au}_{17} \mathrm{Sn}_{3}$ phase shows stability down to $-5{ }^{\circ} \mathrm{C}$ and is known for relatively low modulus and hardness. It is therefore known for a good candidate for thermo-mechanical stress absorber in solder joint.

- The '’ phase, $\mathrm{Au}_{5} \mathrm{Sn}$, has a trigonal structure and is stable below $190^{\circ} \mathrm{C}$. The phase has relatively low modulus but is also brittle in nature. As a result, this $\zeta^{\prime}$ phase is poor candidate as a stress adsorbing layer.

- The $\delta$ phase, AuSn is an equiatomic intermetallic compound. It has a hexagonal crystal structure and melts congruently at $419^{\circ} \mathrm{C}$. It is also relatively high hardness values with high elastic modulus, and is brittle in nature.

- The $\varepsilon$ phase, $\mathrm{AuSn}_{2}$, has an orthorhombic close-packed hexagonal structure. AuSn 2 and AuSn are amongst the most brittle and the hardest IMCs.

- The $\eta$ phase, $\mathrm{AuSn}_{4}$, has an orthorhombic close-packed hexagonal structure. AuSn 4 is more ductile with mechanical properties suitable for solder joint such as lower young modulus and less Vickers hardness.

\section{Experimental Procedure}

\subsection{Preparation of Diffusion Couples}

The following steps were used to prepare the diffusion couple. First step was to roll bulk gold $(99.99 \mathrm{wt} \%)$ and tin (99.97 wt\%) into thin foils of $100 \mu \mathrm{m}$ and $200 \mu \mathrm{m}$ thickness (gold and tin respectively). Each sheet of metal was cut into smaller sheets. The sheets were then sandwiched together to form diffusion couple as shown in Fig. 2 (a). 

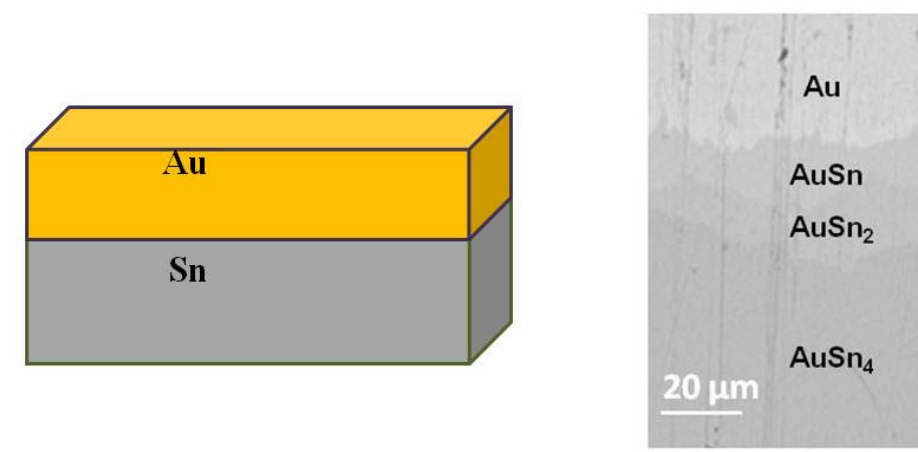

Fig. 2. (a) Left schematic shows Au-Sn diffusion couple prior to heat treatment. After heat treatment, right image (b), is an example of the observed microstructure using electron microscope (back-scattered electron image) after the diffusion couple was isothermally heat treated at $473 \mathrm{~K}$. For calculations, the thicknesses of these layers were measured by using the image area analysis (NIH ImageJ, version 1.6.0) divided by the interface length to obtain averaged thickness values.

\subsection{Heat Treatment}

After preparation diffusion couple, specimens were heat treated in a resistive heating furnace with varying temperature and time. In this study, the two focused temperatures were $453 \mathrm{~K}, 473 \mathrm{~K}$ and $498 \mathrm{~K}$ at 5 different holding times: $1,3,5,7$, and 24 hours. During heat treatment, diffusion couple is loaded with 620 grams of force at all time. Heat treatment conditions are summarized in Table 1. The eutectic temperature of tin-rich composition is $490 \mathrm{~K}$. The specimens that were heat treated at $498 \mathrm{~K}$ represented the effect of temperature above the eutectic temperature but below the melting temperature of elemental tin. The other two sets of specimens were heat treated at $453 \mathrm{~K}$ and $473 \mathrm{~K}$ which were the representation of solid-state diffusion. The holding time revealed microstructure evolution and stability of each phases of Au-Sn intermetallics.

Table 1. Summary of heat treatment conditions isothermal is shown with isothermal temperatures $(\mathrm{K})$ and holding times (h). There are a total of fifteen samples that underwent three isothermal treatments at varied holding time from 1 hour to 24 hours.

\begin{tabular}{|c|c|c|c|c|c|}
\hline Time (h) & 1 & 3 & 5 & 7 & 24 \\
\hline 498 & A1 & A3 & A5 & A7 & A24 \\
\hline 473 & B1 & B3 & B5 & B7 & B24 \\
\hline 453 & C1 & C3 & C5 & C7 & C24 \\
\hline
\end{tabular}

\subsection{Characterization}

After heat treatment, specimens of all conditions were cut into two pieces to investigate their cross-sectional microstructure with optical microscope and scanning electron microscope (Field Emission Scanning Electron Microscope: JEOL JSM-7001F). Energy dispersive x-ray spectroscopy technique was used to monitor the diffusion through the interface. The thickness of layers were extracted using ImageJ software $(\mathrm{NIH}$, version 1.6.0) to calculate area of the intermetallic which was then divided by the interface length in order to obtain averaged thicknesses. 


\section{Results and Discussion}

\subsection{Cross-Sectional Microstructure}

Diffusion couple in as-sandwiched condition is analyzed as a reference to other heat treatment conditions. The interface location and thickness were measured in order to track the interface movement and evolution. New interface between layers of gold and tin is generated and moved because diffusion rates of gold in tin and vice versa are not equal.

The microstructural observation for the case of $473 \mathrm{~K}$ and $498 \mathrm{~K}$ could be shown in Fig. 3 and Fig. 4. First the case of $473 \mathrm{~K}$ is considered in Fig. 3. The $\mathrm{x}$-axis shows the time dimension of the hear treatment at $473 \mathrm{~K}$ while the y-axis shows the thicknesses of the IMCs and phases. The microstructures evolved from Aurich to Sn-rich, and to AuSn. Then later the formation of $\mathrm{AuSn}_{2}$ and $\mathrm{AuSn}_{4}$ followed. In particular it must be noted that $\mathrm{AuSn}_{4}$ was later formed after 7 hours treatment time. In comparison, the case of $498 \mathrm{~K}$, there is a slight change in the IMC formation order.

For the case of $498 \mathrm{~K}$, which is slightly above the eutectic temperature, the phase evolution starts with gold rich and tin rich simultaneously. This could be due to the local rejection of gold atoms from Sn-rich region of the interface because of the extremely limited solid solubility [16]. It was therefore possible to have partial melting locally or the existence of fine gold-rich precipitates inside almost pure tin matrix. AuSn 4 was then formed almost at the same time as $\mathrm{AuSn}_{2}$ while AuSn later grew.

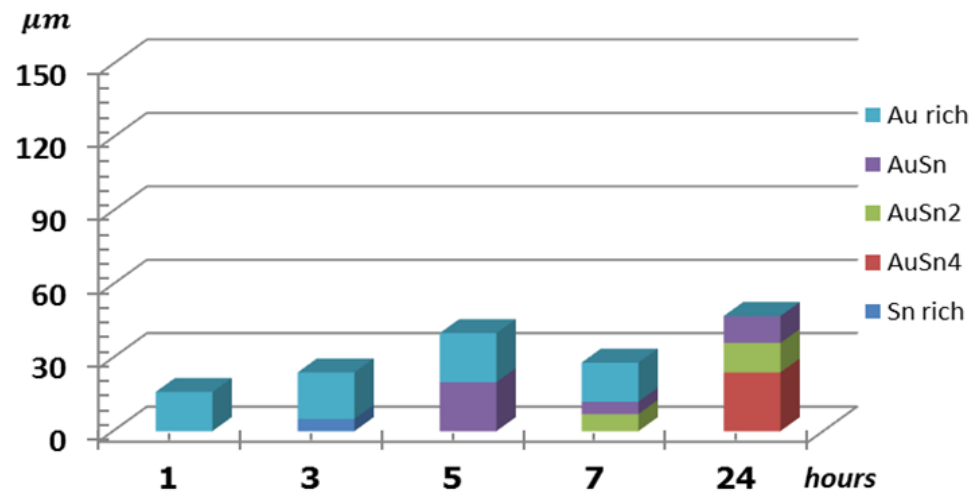

Fig. 3. Microstructure evolution of IMCs for the case heat treatment at $473 \mathrm{~K}$ for 1 to 24 hours. The total thickness of the interface layer was approximately 50 micrometers after 24 hours. The phase evolution started with Au-rich, Sn-rich and then the formation of AuSn. Ultimately $\mathrm{AuSn}_{2}$ and $\mathrm{AuSn}_{4}$ formed.

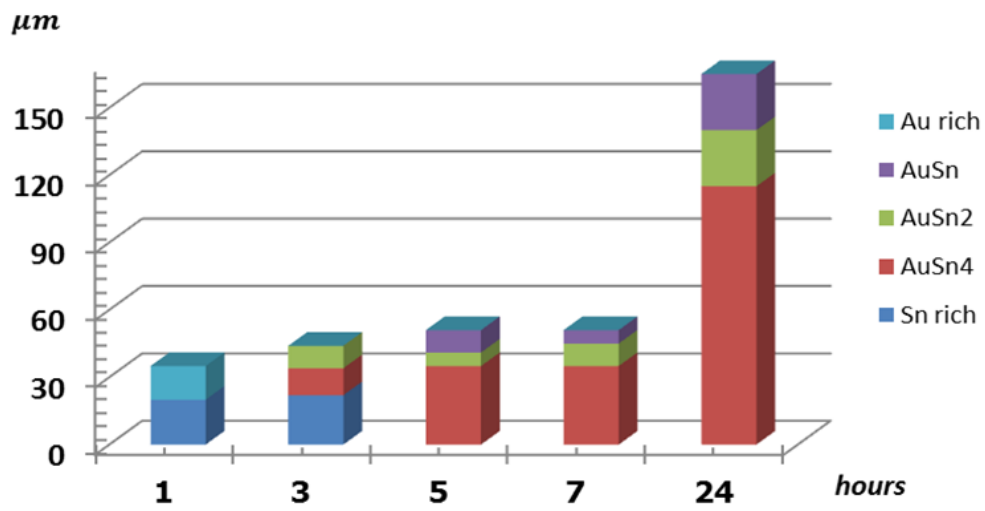

Fig. 4. Microstructure evolution of IMCs for the case heat treatment at $498 \mathrm{~K}$ for 1 to 24 hours. The total thickness of the interface layer was approximately 170 micrometers after 24 hours. The phase evolution is different from the case of $473 \mathrm{~K}$ due to the fact there $498 \mathrm{~K}$ is slightly above eutectic temperature and the mechanism for IMCs could be enhanced by partial melting or dissolution as well as increased diffusion. 


\subsection{Diffusion Assessment}

To make an assessment of the diffusion kinetic, growth behavior of the intermetallic layers was studied by IMC layer thickness measurement method from previous studies [14, 17-19]. The focus is on the comparison specimens which were heat treated at $498 \mathrm{~K}$ and $473 \mathrm{~K}$ because they were comparatively close to the eutectic temperature in the Sn-rich region. The relation between IMCs layer thickness and holding time are shown for the example case of Au-Sn diffusion couple heat treated at $473 \mathrm{~K}$ for 24 hours in Fig. 5. As a function of position of the interface, the graph shows the mole fraction of Au to Sn ranging from 0 to 1 which is a direct indication of the types of IMCs and phases. In this case, $\mathrm{AuSn}_{4}, \mathrm{AuSn}_{2}$, and AuSn were the main three IMCs located in between gold-rich and tin-rich phases. For the growth and overall length of the interface, it was clear that for the case of $473 \mathrm{~K}$, Layer thickness of each IMC phase was measured from EDX line scan data and also confirmed by ImageJ software. The average thickness " $l i$ " of layer " $~ "$ " was evaluated by the equation:

$$
l_{i}=\frac{A_{i}}{w_{i}}
$$

where $A_{i}$ represented the total area of layer $i$ and $w_{i}$ could be measured from the total length of layer $i[14$, 17-19]. For convenience, designation of $\mathrm{AuSn}, \mathrm{AuSn}_{2}$, and $\mathrm{AuSn}_{4}$ IMC layers were designated with the subscripts of $\mathrm{i}=1,2$, and 4 , respectively. Moreover, the $l_{1}$ and $l_{2}$ were evaluated as the summation $l_{s}$ because the AuSn layer did not explicitly segregate from $\mathrm{AuSn}_{2}$ layer. Furthermore, the total layer thickness $l$ is the summation of all IMC layers; $l_{s}$ and $l_{4}$ because $l=l_{s}$ and $l_{4}$. After the average thickness $l_{i}$ of all IMC layers for heat treated at $498 \mathrm{~K}$ and $473 \mathrm{~K}$ were obtained, $n$ and $k_{i}$ values were calculated by the least-squares method based on the following equation:

$$
l_{i}=k_{i}\left(\frac{t}{t_{0}}\right)^{n}
$$

where $t_{0}$ was unity time, 1 second and the ratio $t / t_{0}$ is dimensionless [14,17-19]. The proportional coefficient $k_{i}$ has the same unit dimension as the layer thickness $l_{i}$. In addition, the exponent $n$ is dimensionless. The values of $k$ and $n$ were estimated by the least-squares method as show in Fig. 6 and Fig. 7 representing the case of $473 \mathrm{~K}$ and $498 \mathrm{~K}$ heat treatment profiles. In Fig. 6 and Fig. 7, the ordinate and the abscissa indicate the logarithm of IMCs layer thickness and the logarithm of holding time t, respectively. By assuming a monotonically increasing trend, the proportional coefficient $k_{L}$ and exponent $n$ were determined to be: (a) $k_{L}$ $=2.99 \times 10^{-8} \mathrm{~m}$ and $n=0.68$ for $473 \mathrm{~K}$ heat treatment, and (b) $k_{L}=6.01 \times 10^{-8} \mathrm{~m}$ and $n=0.69$ for $498 \mathrm{~K}$ heat treatment. The individual $k$ and $n$ values for $\mathrm{L}_{4}$ and $\mathrm{L}_{\mathrm{s}}$ could be found as insets in Fig. 6 and Fig. 7. 
Au/Sn 473K 24h

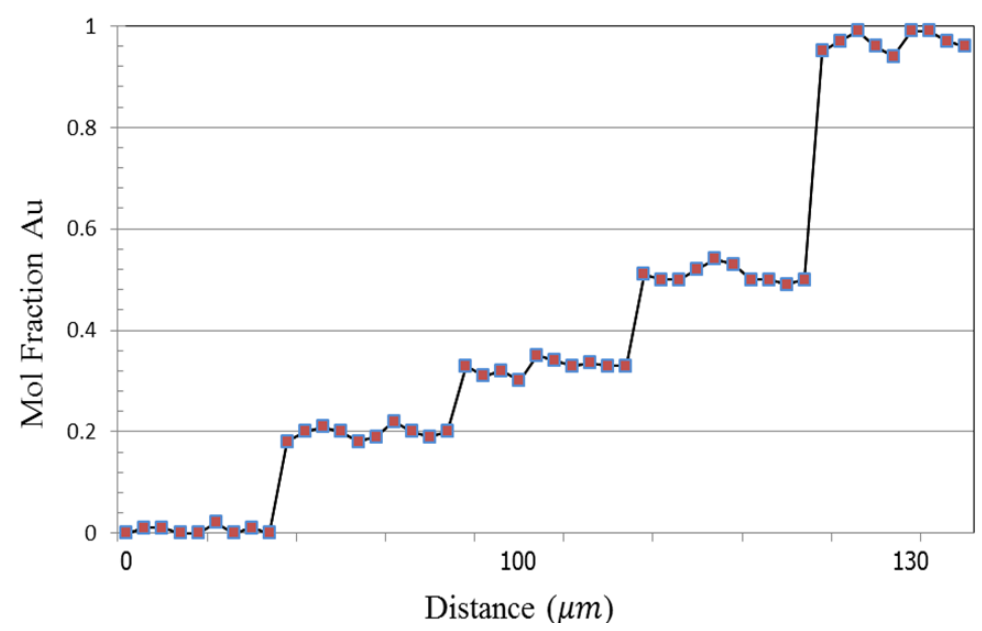

Fig. 5. Mole fraction Au to Sn from EDX line scan is shown as a function of distance from the interfaces. Pure $\mathrm{Sn}$ is located at distant marker 0, while pure gold is located at the far right of the graph. There are 3 IMCs found in this case which are: $\mathrm{AuSn}_{4}, \mathrm{AuSn}_{2}$, and AuSn.
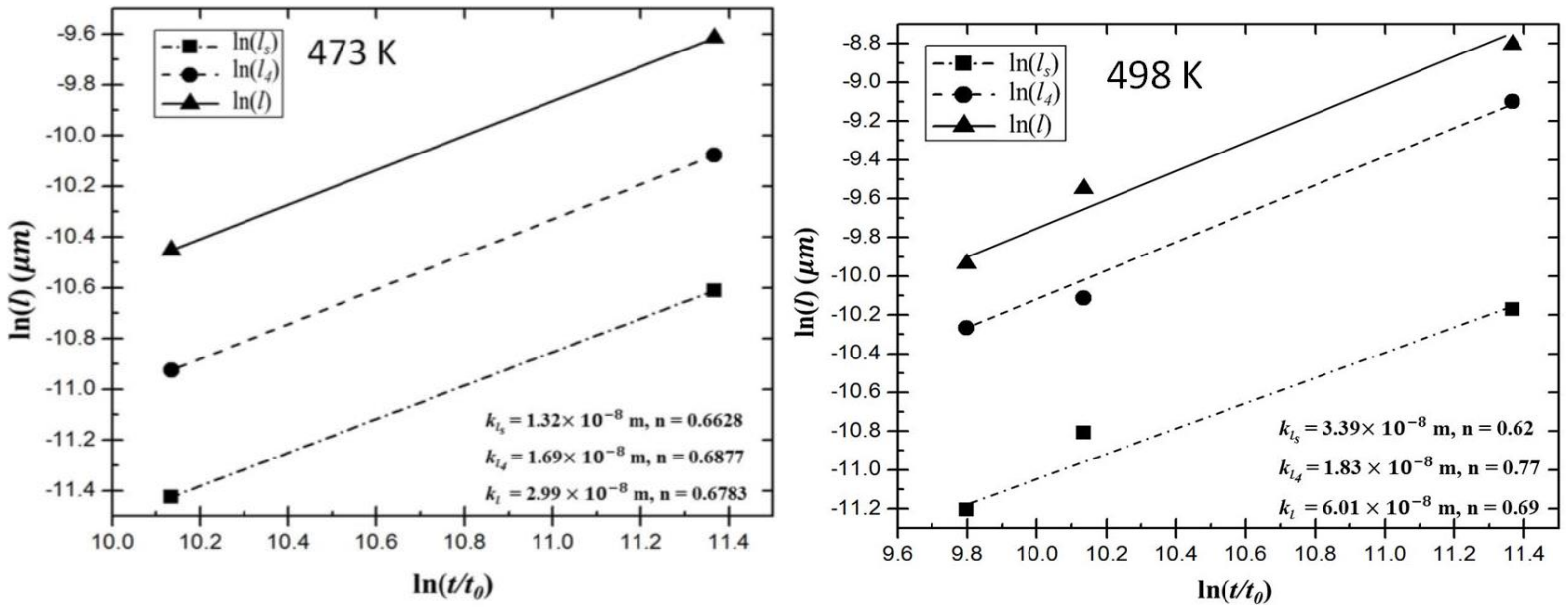

Fig. $6 \& 7$. The graphs show the values of $l$ as a function of $t / t_{0}$ through a logarithm relationship. The values of $k$ and $n$ could then be obtained from the slope and the $\mathrm{y}$-intersect. The graphs are the responses for the case of $473 \mathrm{~K}$ and $498 \mathrm{~K}$ for slightly below and above the eutectic temperature of tin-rich composition. The $k$ values are shown for the case of AuSn and $\mathrm{AuSn}_{2}\left(k_{l l}\right), \mathrm{AuSn}_{4}\left(k_{i}\right)$ and all layers $\left(k_{i}\right)$.

\subsection{Rate-Controlling Processes}

The exponent $n$ is typically greater than 0.5 . At $n=0.5$, the relationship is parabolic which is common for volume diffusion and diffusion controlled kinetic. In particular if the process involves a precipitation process in a primary untransformed matrix, then the relationship is usually parabolic. If $n$ is equal to 1.0 , the relationship becomes linear and the growth of the IMC is controlled by the reaction at the interface. If $n$ value is slightly below 0.5 then grain boundary diffusion might contribute to the rate controlling process [14, 20]. In our case, the calculated $n$ is between $0.62-0.77$ which implies that the kinetics of IMC formations experiment are controlled by both diffusion and intermetallic reaction. Especially, at high temperature, the $n$ power was found to be larger because of the partial melting of Sn-rich region. Then gold atoms were quickly dissolute into molten Sn-rich liquid. 


\section{Conclusions}

For heat treatment at below eutectic temperature, the IMC formation was governed by the solid-state interdiffusion of $\mathrm{Au}$ and Sn. The first IMC phase that formed between Au/Sn interface was AuSn. The equiatomic AuSn compound is preferred because both $\mathrm{Sn}$ and Au possessed similar electronic valence. Over longer period of time, as Au diffused into $\mathrm{Sn}$ faster, $\mathrm{AuSn}_{2}$ formed on the AuSn/Sn interface as more Au diffused into Sn-rich region. AuSn 4 was the last phase that formed on $\mathrm{AuSn}_{2} / \mathrm{Sn}$ interface.

For heat treatment at temperature slightly above the eutectic temperature, partial melting of $S n$ took place; consequently, Au dissolved into molten Sn-rich localized region resulting in an early formation of AuSn 4 phase along with AuSn. Subsequently, $\mathrm{AuSn}_{2}$ formed between AuSn 4 /AuSn interface.

For industrial applications, soldering and annealing parameters are key factors leading to reliable solder joints. In real life operation, an increase in temperature with respect to time could cause the layers of different IMC phases to grow at different rates. Two distinguished mechanisms for rate controlling processes were found even if the temperature difference is only $25 \mathrm{~K}$. Combined thickness of AuSn and AuSn 2 phases is less than thickness that of only $\mathrm{AuSn}_{4}$ phase. At the beginning of heat treatment, growth rate of $\mathrm{AuSn}_{4}$ was also higher than that of either $\mathrm{AuSn}$ or $\mathrm{AuSn}_{2}$. However, growth rate of $\mathrm{AuSn}_{4}$ decrease and is slower than that of $\mathrm{AuSn}$ and $\mathrm{AuSn}_{2}$ after undergoing the annealing process in the range of 24 hours. Interdiffusion layers of IMCs could be clearly observed as the specimens were heat treated for over 7 hours. Similarly, phase stability can be also seen if specimens were heat treated for longer time. AuSn, AuSn $n_{2}$, and $\mathrm{AuSn}_{4}$ are the IMC phases from the Au-Sn system that were proven relatively stable at room temperature. The research work could be adapted to the soldering processes in both jewelry and electronic industries. The insights to the mechanisms suggest two possibilities for bonding process at different temperatures. For slightly above eutectic, the diffusion process is faster and preferred as the interface microstructure was uniformed and controllable. The longevity and reliability of soldered joint should also be further investigated for shearing, cyclical fatigue and failure modes.

\section{References}

[1] G. S. Matijasevic, C. C. Lee, and C. Y. Wang, "Au-Sn alloy phase diagram and properties related to its use as a bonding medium," Thin Solid Films, vol. 223, no. 2, pp. 276-287, 1993.

[2] R. R. Chromik, D.-N. Wang, A. Shugar, L. Limata, M. R. Notis, and R. P. Vinci, "Mechanical properties of intermetallic compounds in the Au-Sn system," Journal of Materials Research, vol. 20, no. 8, pp. 2161 2172, 2005.

[3] T. A. Tollefsen, A. Larsson, O. M. Løvvik, and K. Aasmundtveit, "Au-Sn SLID Bonding-Properties and Possibilities," Metallurgical and Materials Transactions B, vol. 43, no. 2, pp. 397-405, 2012.

[4] C. Hillman, "Processing and reliability issues for eutectic AuSn solder joints," in 41st International Symposium on Microelectronics (IMAPS 2008) Proceedings, 2008, pp. 909-916.

[5] T. Tollefsen, A. Larsson, M. M. V. Taklo, A. Neels, X. Maeder, K. Høydalsvik, D. Breiby, and K. Aasmundtveit, "Au-Sn SLID bonding: A reliable HT interconnect and die attach technology," Metallurgical and Materials Transactions B, vol. 44, no. 2, pp. 406-413, 2013.

[6] W. Reinert and P. Merz, "Metallic alloy seal bonding," in Handbook of Silicon Based MEMS Materials and Technologies, Boston: William Andrew, 2010, ch. 34, pp. 533-542.

[7] M. L. Huang, Y. Liu, and J. X. Gao, "Interfacial reaction between Au and Sn films electroplated for LED bumps," Journal of Materials Science: Materials in Electronics, vol. 22, no. 2, pp. 193-199, 2011.

[8] J. W. Yoon, H. S. Chun, J. M. Koo, and S. B. Jung, "Au-Sn flip-chip solder bump for microelectronic and optoelectronic applications," Microsystem Technologies, vol. 13, no. 11-12, pp. 1463-1469, 2007.

[9] J. W. Yoon, H. S. Chun, and S. B. Jung, "Reliability evaluation of Au-20Sn flip chip solder bump fabricated by sequential electroplating method with Sn and Au," Materials Science and Engineering: A, vol. 473, no. 1-2, pp. 119-125, 2008.

[10] J. Wei, "A new approach of creating Au-Sn solder bumps from electroplating," Crystal Research and Technology, vol. 41, no. 2, pp. 150-153, 2006.

[11] A. Dębski, W. Gąsior, Z. Moser, and R. Major, "Enthalpy of formation of intermetallic phases from the Au-Sn system," Journal of Alloys and Compounds, vol. 491, no. 1-2, pp. 173-177, 2010.

[12] C. C. Lee, C. Y. Wang, and G. S. Matijasevic, "A new bonding technology using gold and tin multilayer composite structures," IEEE Transactions, vol. 14, no. 2, pp. 407 - 412, 1991. 
[13] G. Elger, M. Hutter, H. Oppermann, R. Aschenbrenner, H. Reichl, and E. Jäger, "Development of an assembly process and reliability investigations for flip-chip LEDs using the AuSn soldering," Microsystem Technologies, vol. 7, no. 5-6, pp. 239-243, 2002.

[14] T. Yamada, K. Miura, M. Kajihara, N. Kurokawa, and K. Sakamoto, "Kinetics of reactive diffusion between $\mathrm{Au}$ and $\mathrm{Sn}$ during annealing at solid-state temperatures," Materials Science and Engineering: $A$, vol. 390, no. 1-2, pp. 118-126, 2005.

[15] Y. K. Lee, Y. H. Ko, J. K. Kim, C. W. Lee, and S. Yoo, "The effect of intermetallic compound evolution on the fracture behavior of Au stud bumps joined with Sn-3.5Ag solder," Electronic Materials Letters, vol. 9, no. 1, pp. 31-39, 2013.

[16] J. Ciulik and M. R. Notis, "The Au-Sn phase diagram," Journal of Alloys and Compounds, vol. 191, no. 1, pp. 71-78, 1993.

[17] M. Mita, K. Miura, T. Takenaka, M. Kajihara, N. Kurokawa, and K. Sakamoto, "Effect of Ni on reactive diffusion between $\mathrm{Au}$ and $\mathrm{Sn}$ at solid-state temperatures," Materials Science and Engineering: B, vol. 126, no. 1, pp. 37-43, 2006.

[18] T. Yamada, K. Miura, M. Kajihara, N. Kurokawa, and K. Sakamoto, "Formation of intermetallic compound layers in $\mathrm{Sn} / \mathrm{Au} / \mathrm{Sn}$ diffusion couple during annealing at $433 \mathrm{~K}$," Journal of Materials Science, vol. 39, no. 7, pp. 2327-2334, 2004.

[19] Y. Yato and M. Kajihara, "Kinetic features of reactive diffusion between Sn-5Au alloy and Ni at solidstate temperatures," Materials Transactions, vol. 47, no. 9, pp. 2277-2284, 2006.

[20] T. Takenaka, M. Kajihara, N. Kurokawa, and K. Sakamoto, "Reactive diffusion between Ag-Au alloys and Sn at solid-state temperatures," Materials Science and Engineering: A, vol. 427, no. 1-2, pp. 210-222, 2006. 